\title{
Biperiodicity in neutral-type delayed difference neural networks
}

Zhenkun Huang $^{1 *}$ and Youssef N Raffoul ${ }^{2}$

* Correspondence:

hzk974226@jmu.edu.cn

'School of Science, Jimei

University, Xiamen 361021, P. R.

China

Full list of author information is

available at the end of the article

\section{Abstract}

In this article we employ Krasnoselskii's fixed point theorem to obtain new biperiodicity criteria for neutral-type difference neural networks with delays. It is shown that the neutral-type term can leads to biperiodicity results. That is coexistence of a positive periodic sequence solution and its anti-sign periodic sequence solution. We illustrate our novel approach the biperiodicity dynamics of biperiodicity for neutral-type delay difference neural networks by two computer numerical examples.

Mathematics Subject Classification 2010: 39A23; 39A10.

Keywords: difference neural networks, biperiodicity, neutral-type, delayed

\section{Introduction}

It is well known that neural networks with delays have a rich dynamical behavior that have been recently investigated by Huand and $\mathrm{Li}$ [1] and the references therein. It is naturally important that such systems should contain some information regarding the past rate of change since they effectively describe and model the dynamic of the application of neural networks [2-4]. As a consequence, scholars and researchers have paid more attention to the stability of neural networks that are described by nonlinear delay differential equations of the neutral type (see [4-8])

$$
\begin{aligned}
\dot{u}_{i}(t) & =-a_{i}(t) u_{i}(t)+\sum_{j=1}^{m} b_{i j}(t) g_{j}\left(u_{j}(t)\right)+\sum_{j=1}^{m} c_{i j} \dot{u}_{j}(t-\tau) \\
& +\sum_{j=1}^{m} d_{i j}(t) g_{j}\left[\int_{-\infty}^{t} h_{j}(t-s) u_{j}(s) d s\right]+I_{i}(t), i \in \mathcal{N}:=\{1,2, \cdots, m\}
\end{aligned}
$$

Cheng et al. first investigated the globally asymptotic stability of a class of neutraltype neural networks with delays [6]. Delay-dependent criterion has been attained in [5] by using Lyapunov stability theory and linear matrix inequality. Recently a conservative robust stability criteria for neutral-type networks with delays are proposed in [4] by using a new Lyapunov-Krasovskii functional and a novel series compensation technique. For more relative results, we can refer to $[4,7]$ and references cited therein.

Difference equations or discrete-time analogs of differential equations can preserve the convergence dynamics of their continuous-time counterparts in some degree [9]. So, due to its usage in computer simulations and applications, these discrete-type or

(c) 2012 Huang and Raffoul; licensee Springer. This is an Open Access article distributed under the terms of the Creative Commons Attribution License (http://creativecommons.org/licenses/by/2.0), which permits unrestricted use, distribution, and reproduction in any medium, provided the original work is properly cited. 
difference networks have been deeply discussed by the authors of [10-15] and extended to periodic or almost periodic difference neural systems [16-21].

However, few papers deal with multiperiodicity of neutral-type difference neural networks with delays. Stimulated by the articles [22,23], in this article, we should consider corresponding neutral-type difference version of (1.1) as follows:

$$
\begin{aligned}
u_{i}(n+1)=a_{i}(n) u_{i}(n) & +\sum_{j=1}^{m} c_{i j} \Delta u_{j}(n-\tau)+\sum_{j=1}^{m} b_{i j}(n) g_{j}\left(u_{j}(n)\right) \\
& +\sum_{j=1}^{m} d_{i j}(n) g_{j}\left[\sum_{v=1}^{\infty} h_{j}(v) u_{j}(n-v)\right]+I_{i}(n),
\end{aligned}
$$

where $i \in \mathcal{N}:=\{1,2, \ldots, m\}$. Our main aim is to study biperiodicity of the above neutral-type difference neural networks. Some new criteria for coexistence of a periodic sequence solution and anti-sign periodic one of (1.2) have been derived by using Krasnoselskii's fixed point theorem. Our results are completely different from monoperiodicity existing ones in [16-20].

The rest of this article is organized as follows. In Section 2, we shall make some preparations by giving some lemmas and Krasnoselskii's fixed point theorem. In Section 3, we gives new criteria for biperiodicity of (1.2). Finally, two numerical examples are given to illustrate our results.

\section{Preliminaries}

We begin this section by introducing some notations and some lemmas. Let $\mathcal{S}_{T}$ be the set of all real $T$-periodic sequences defined on $\mathbb{Z}$, where $T$ is an integer with $T \geq 1$. Then $\mathcal{S}_{T}$ is a Banach space when it is endowed with the norm

$$
\|u\|=\max _{i \in \mathcal{N}}\left\{\sup _{s \in[0, T]_{\mathbb{Z}}}\left|u_{i}(s)\right|\right\} .
$$

Denote $[a, b]_{\mathbb{Z}}:=\{a, a+1, \ldots, b\}$, where $a, b \in \mathbb{Z}$ and $a \leq b$. Let $C\left((-\infty, 0]_{\mathbb{Z}}, \mathbb{R}^{m}\right)$ be the set of all continuous and bounded functions $\psi(s)=\left(\psi_{1}(s), \psi_{2}(s), \ldots, \psi_{m}(s)\right)^{T}$ mapping $(-\infty, 0]_{Z}$ into $\mathbb{R}^{m}$. For any given $\psi \in C\left((-\infty, 0]_{Z}, \mathbb{R}^{N}\right)$, we denote by $\{u(n ; \psi)\}$ the sequence solution of system (1.2). Next, we present the basic assumptions:

- Assumption $\left(H_{1}\right)$ : Each $a_{i}(\cdot), b_{i j}(\cdot), d_{i j}(\cdot)$, and $I_{i}(\cdot)$ are $T$-periodic functions defined on $\mathbb{Z}, 0<a_{i}(n)<1$. The activation $g_{j}(\cdot)$ is strictly increasing and bounded with $-g_{j}^{\natural}=\lim _{v \rightarrow-\infty} g_{j}(v)<g_{j}(v)<\lim _{v \rightarrow+\infty} g_{j}(v)=g_{j}^{\natural}$ for all $v \in \mathbb{R}$. The kernel $h_{j}: \mathbb{N}$

$\rightarrow \mathbb{R}^{+}$is a bounded sequence with $\sum_{v=1}^{\infty} h_{j}(v)=1$, where $i, j \in \mathcal{N}$.

For each $i \in \mathcal{N}$ and any $n \in \mathbb{Z}$, we let

$$
G_{i}(n, p)=\prod_{s=p+1}^{n+T-1} a_{i}(s)\left[1-\prod_{s=n}^{n+T-1} a_{i}(s)\right]^{-1}, p \in[n, n+T-1]
$$

Since $0<a_{i}(n)<1$ for all $n \in[0, T-1]$, each $G_{i}(n, p)$ is not zero and

$$
\begin{aligned}
& m_{i}:=\min \left\{G_{i}(n, p): n \geq 0, p \leq T\right\}=G_{i}(n, n)=G_{i}(0,0)>0, \\
& M_{i}:=\max \left\{G_{i}(n, p): n \geq 0, p \leq T\right\}=G_{i}(n, n+T-1)=G_{i}(0, T-1)>0 .
\end{aligned}
$$


Lemma 2.1. For each $i \in \mathbb{N}$ and $\forall p \in \mathbb{Z}^{+}$,

$$
\mathscr{P}\left[\prod_{s=0}^{p-1} a_{i}^{-1}(s)\right] \Delta u_{i}(p-\tau)+u_{i}(p-\tau) \Delta\left[\prod_{s=0}^{p-1} a_{i}^{-1}(s)\right]=\Delta\left[\prod_{s=0}^{p-1} a_{i}^{-1}(s) u_{i}(p-\tau)\right]
$$

holds for any sequence solution $\{u(n)\}$ of (1.2), where, $\mathscr{S}$ is a shift operator defined as $i \in \mathcal{N}$ for $i \in \mathcal{N}$ and $p \in \mathbb{Z}^{+}$.

Proof.

$$
\begin{aligned}
& \mathscr{S}\left[\prod_{s=0}^{p-1} a_{i}^{-1}(s)\right] \Delta u_{i}(p-\tau)+u_{i}(p-\tau) \Delta\left[\prod_{s=0}^{p-1} a_{i}^{-1}(s)\right] \\
& =\prod_{s=0}^{p} a_{i}^{-1}(s)\left[u_{i}(p+1-\tau)-u_{i}(p-\tau)\right]+u_{i}(p-\tau)\left[\prod_{s=0}^{p} a_{i}^{-1}(s)-\prod_{s=0}^{p-1} a_{i}^{-1}(s)\right] \\
& =\prod_{s=0}^{p} a_{i}^{-1}(s) u_{i}(p+1-\tau)-\prod_{s=0}^{p-1} a_{i}^{-1}(s) u_{i}(p-\tau) \\
& =\triangle\left[\prod_{s=0}^{p-1} a_{i}^{-1}(s) u_{i}(p-\tau)\right] .
\end{aligned}
$$

The proof is complete.

Lemma 2.2. Assume that $\left(H_{1}\right)$ hold. Any sequence $\{u(n)\} \in \mathcal{S}_{T}^{m}:=\underbrace{\mathcal{S}_{T} \times \mathcal{S}_{T} \times \cdots \times \mathcal{S}_{T}}_{m}$ is a solution of (1.2) if and only if

$$
\begin{aligned}
u_{i}(n) & =\sum_{j=1}^{m} c_{i j} u_{j}(n-\tau)+\sum_{p=n}^{n+T-1} G_{i}(n, p)\left[\sum_{j=1}^{m} b_{i j}(p) g_{j}\left(u_{j}(p)\right)\right. \\
& \left.+\sum_{j=1}^{m} d_{i j}(p) g_{j}\left(\sum_{v=1}^{\infty} h_{j}(v) u_{j}(p-v)\right)+I_{i}(p)-\sum_{j=1}^{m} c_{i j} u_{j}(p-\tau)\left(1-a_{i}(p)\right)\right],
\end{aligned}
$$

where $G_{i}(n, p)$ is defined by (2.1) for $i \in \mathcal{N}$ and $p \in \mathbb{Z}^{+}$.

Proof. Rewrite (1.2) as

$$
\begin{aligned}
\Delta\left[u_{i}(n) \prod_{s=0}^{n-1} a_{i}^{-1}(s)\right] & =\left[\sum_{j=1}^{m} c_{i j} \Delta u_{j}(n-\tau)+\sum_{j=1}^{m} b_{i j}(n) g_{j}\left(u_{j}(n)\right)\right. \\
& \left.+\sum_{j=1}^{m} d_{i j}(n) g_{j}\left(\sum_{v=1}^{\infty} h_{j}(v) u_{j}(n-v)\right)+I_{i}(n)\right] \prod_{s=0}^{n} a_{i}^{-1}(s),
\end{aligned}
$$

where $i \in \mathcal{N}$ and $n \in \mathbb{Z}^{+}$. Summing (2.3) from $n$ to $n+T$ - 1, we obtain

$$
\begin{aligned}
\sum_{p=n}^{n+T-1} \Delta\left[u_{i}(p) \prod_{s=0}^{p-1} a_{i}^{-1}(s)\right] & =\sum_{p=n}^{n+T-1}\left[\sum_{j=1}^{m} c_{i j} \Delta u_{j}(p-\tau)+\sum_{j=1}^{m} b_{i j}(p) g_{j}\left(u_{j}(p)\right)\right. \\
& \left.+\sum_{j=1}^{m} d_{i j}(p) g_{j}\left(\sum_{v=1}^{\infty} h_{j}(v) u_{j}(p-v)\right)+I_{i}(p)\right] \prod_{s=0}^{p} a_{i}^{-1}(s) .
\end{aligned}
$$


That is,

$$
\begin{aligned}
& u_{i}(n+T) \prod_{s=0}^{n+T-1} a_{i}^{-1}(s)-u_{i}(n) \prod_{s=0}^{n-1} a_{i}^{-1}(s) \\
= & \sum_{p=n}^{n+T-1}\left[\sum_{j=1}^{m} c_{i j} \Delta u_{j}(p-\tau)+\sum_{j=1}^{m} b_{i j}(p) g_{j}\left(u_{j}(p)\right)\right. \\
+ & \left.\sum_{j=1}^{m} d_{i j}(p) g_{j}\left(\sum_{v=1}^{\infty} h_{j}(v) u_{j}(p-v)\right)+I_{i}(p)\right] \prod_{s=0}^{p} a_{i}^{-1}(s) .
\end{aligned}
$$

Since $u_{i}(n+T)=u_{i}(n)$, we obtain

$$
\begin{aligned}
& u_{i}(n)\left[\prod_{s=0}^{n+T-1} a_{i}^{-1}(s)-\prod_{s=0}^{n-1} a_{i}^{-1}(s)\right] \\
= & \sum_{p=n}^{n+T-1}\left[\sum_{j=1}^{m} c_{i j} \Delta u_{j}(p-\tau)+\sum_{j=1}^{m} b_{i j}(p) g_{j}\left(u_{j}(p)\right)\right. \\
+ & \left.\sum_{j=1}^{m} d_{i j}(p) g_{j}\left(\sum_{v=1}^{\infty} h_{j}(v) u_{j}(p-v)\right)+I_{i}(p)\right] \prod_{s=0}^{p} a_{i}^{-1}(s) .
\end{aligned}
$$

It follows from Lemma 2.1 that

$$
\begin{aligned}
& \sum_{p=n}^{n+T-1} \sum_{j=1}^{m} c_{i j} \Delta u_{j}(p-\tau) \prod_{s=0}^{p} a_{i}^{-1}(s)=\sum_{p=n}^{n+T-1} \sum_{j=1}^{m} c_{i j} \Delta u_{j}(p-\tau) \mathscr{S}\left[\prod_{s=0}^{p-1} a_{i}^{-1}(s)\right] \\
= & \sum_{p=n}^{n+T-1} \sum_{j=1}^{m} c_{i j}\left\{\Delta\left[u_{j}(p-\tau) \prod_{s=0}^{p-1} a_{i}^{-1}(s)\right]-u_{j}(p-\tau) \Delta\left[\prod_{s=0}^{p-1} a_{i}^{-1}(s)\right]\right\} \\
= & \sum_{j=1}^{m} c_{i j}\left\{\sum_{p=n}^{n+T-1} \Delta\left[u_{j}(p-\tau) \prod_{s=0}^{p-1} a_{i}^{-1}(s)\right]\right\}-\sum_{p=n}^{n+T-1} \sum_{j=1}^{m} c_{i j} u_{j}(p-\tau) \Delta\left[\prod_{s=0}^{p-1} a_{i}^{-1}(s)\right] \\
= & \sum_{j=1}^{m} c_{i j} u_{j}(n-\tau)\left[\prod_{s=0}^{n+T-1} a_{i}^{-1}(s)-\prod_{s=0}^{n-1} a_{i}^{-1}(s)\right]-\sum_{p=n}^{n+T-1} \sum_{j=1}^{m} c_{i j} u_{j}(p-\tau) \Delta\left[\prod_{s=0}^{u-1} a_{i}^{-1}(s)\right] .
\end{aligned}
$$

Therefore, one gets from (2.4) that

$$
\begin{aligned}
& u_{i}(n)\left[\prod_{s=0}^{n+T-1} a_{i}^{-1}(s)-\prod_{s=0}^{n-1} a_{i}^{-1}(s)\right]=\sum_{j=1}^{m} c_{i j} u_{j}(n-\tau)\left[\prod_{s=0}^{n+T-1} a_{i}^{-1}(s)-\prod_{s=0}^{n-1} a_{i}^{-1}(s)\right] \\
- & \sum_{p=n}^{n+T-1} \sum_{j=1}^{m} c_{i j} u_{j}(p-\tau)\left(1-a_{i}(p)\right) \prod_{s=0}^{p} a_{i}^{-1}(s)+\sum_{p=n}^{n+T-1}\left[\sum_{j=1}^{m} b_{i j}(p) g_{j}\left(u_{j}(p)\right)\right. \\
& \left.+\sum_{j=1}^{m} d_{i j}(p) g_{j}\left(\sum_{v=1}^{\infty} h_{j}(v) u_{j}(p-v)\right)+I_{i}(p)\right] \prod_{s=0}^{p} a_{i}^{-1}(s) .
\end{aligned}
$$

Dividing both sides of the above equation by $\prod_{s=0}^{n+T-1} a_{i}^{-1}(s)-\prod_{s=0}^{n-1} a_{i}^{-1}(s)$ completes the proof.

In what follows, we state Krasnoselskii's theorem.

Lemma 2.3. Let $\mathcal{M}$ be a closed convex nonempty subset of a Banach space $(\mathcal{B},\|\cdot\|)$. 
Suppose that $C$ and $B$ map $\mathcal{M}$ into $\mathcal{B}$ such that

(i) $x, y \in \mathcal{M}$ implies that $C x+B y \in \mathcal{M}$,

(ii) $C$ is continuous and $C \mathcal{M}$ is contained in a compact set and

(iii) $B$ is a contraction mapping.

Then there exists a $z \in \mathcal{M}$ with $z=C z+B z$.

\section{Biperiodicity of neutral-type difference networks}

Due to the introduction of the neutral term neutral $\sum_{j=1}^{m} c_{i j}$, we must construct two closed convex subsets $\mathscr{B}^{L}$ and $\mathscr{B}^{R}$ in $\mathcal{S}_{T}^{m}$, which necessitate the use of Krasnoselskii's fixed point theorem. As a consequence, we are able to derive the new biperiodicity criteria for (1.2). That is there exists a positive $T$-periodic sequence solution in $\mathscr{B}^{R}$ and an anti-sign $T$-periodic sequence solution in $\mathscr{B}^{L}$. Next, for the case $c_{i j} \geq 0$, we present the following assumption:

- Assumption $\left(H_{2}\right)$ : For each $i, j \in \mathcal{N}, c_{i j} \geq 0, b_{i i}(n)>0$ and $0<\hat{c}_{i}:=\sum_{j=1}^{m} c_{i j}<1$, $g_{j}(\cdot)$ satisfies $g_{j}(-v)=-g_{j}(v)$ for all $v \in \mathbb{R}$. Moreover, there exist constants $\alpha>0$ and $\beta>0$ with $\alpha<\beta$ such that for all $i \in \mathcal{N}$

$$
\left.\begin{array}{l}
{\left[-\frac{1-\hat{c}_{i}}{m_{i} T} \alpha+b_{i i}(n) g_{i}(\alpha)\right]-\left(1-a_{i}(n)\right) \hat{c}_{i} \beta>P_{i,}} \\
-\left[-\frac{1-\hat{c}_{i}}{M_{i} T} \beta+b_{i i}(n) g_{i}(\beta)\right]+\left(1-a_{i}(n)\right) \hat{c}_{i} \alpha>p_{i},
\end{array}\right\} \forall n \in \mathbb{Z}
$$

where

$$
P_{i}:=\sup _{n \in \mathbb{Z}}\left\{\sum_{j \neq i}\left|b_{i j}(n)\right| g_{j}^{\natural}+\sum_{j=1}^{m}\left|d_{i j}(n)\right| g_{j}^{\natural}+\left|I_{i}(n)\right|\right\}, i \in \mathcal{N}
$$

Construct two subsets of $\mathcal{S}_{T}$ as follows:

$$
\mathscr{B}_{l}:=\left\{w \in \mathcal{S}_{T} \mid-\beta \leq w(n) \leq-\alpha\right\}, \quad \mathscr{B}_{r}:=\left\{w \in \mathcal{S}_{T} \mid \alpha \leq w(n) \leq \beta\right\} .
$$

Obviously, ${ }^{L}:=\underbrace{\mathscr{B}_{l} \times \mathscr{B}_{l} \cdots \times \mathscr{B}_{l}}_{m}$ and $\mathscr{B}^{R}:=\underbrace{\mathscr{B}_{r} \times \mathscr{B}_{r} \cdots \times \mathscr{B}_{r}}_{m}$ are two closed convex subsets of Banach space $\mathcal{S}_{T}^{m}$. Define the map $B^{\Sigma}: \mathscr{B}^{\Sigma} \rightarrow \mathcal{S}_{T}^{m}$ by

$$
\left(B^{\Sigma} u\right)_{i}(n)=\sum_{j=1}^{m} c_{i j} u_{j}(n-\tau), \quad i \in \mathcal{N}
$$

and the map $C^{\Sigma}: \mathscr{B}^{\Sigma} \rightarrow \mathcal{S}_{T}^{m}$ by

$$
\begin{aligned}
\left(C^{\Sigma} u\right)_{i}(n) & =\sum_{p=n}^{n+T-1} G_{i}(n, p)\left[\sum_{j=1}^{m} b_{i j}(p) g_{j}\left(u_{j}(p)\right)\right. \\
& -\sum_{j=1}^{m} c_{i j} u_{j}(p-\tau)\left(1-a_{i}(p)\right) \\
& \left.+\sum_{j=1}^{m} d_{i j}(p) g_{j}\left(\sum_{v=1}^{\infty} h_{j}(v) u_{j}(p-v)\right)+I_{i}(p)\right], \quad i \in \mathcal{N}
\end{aligned}
$$


where $\Sigma=R$ or $L$. Due to the fact $0<\hat{c}_{i}<1, B^{\Sigma}$ defines a contraction mapping.

Proposition 3.1. Under the basic assumptions $\left(H_{1}\right)$ and $\left(H_{2}\right)$, for each $\Sigma$, the operator $C^{\Sigma}$ is completely continuous on $\mathscr{B} \Sigma$.

Proof. For any given $\Sigma$ and $u \mathscr{B} \Sigma$, we have two cases for the estimation of $\left(C^{\Sigma} u\right)_{i}(n)$.

- Case 1: As $\Sigma=R$ and $u \in \mathscr{B}^{R}, u_{i}(n) \in[\alpha, \beta]$ holds for each $i \in \mathcal{N}$ and all $n \in \mathbb{Z}$. It follows from $(3.1)$ and $\left(H_{2}\right)$ that

$$
\begin{aligned}
\left(C^{R} u\right)_{i}(n) & \leq \sum_{p=n}^{n+T-1} G_{i}(n, p)\left[b_{i i}(p) g_{i}(\beta)+\sum_{j \neq i}\left|b_{i j}(p)\right| g_{j}^{\natural}-\sum_{j=1}^{m} c_{i j} \alpha\left(1-a_{i}(p)\right)\right. \\
& \left.+\sum_{j=1}^{m}\left|d_{i j}(p)\right| g_{j}^{\natural}+\left|I_{i}(p)\right|\right] \\
& \leq \sum_{p=n}^{n+T-1} G_{i}(n, p)\left[-\hat{c}_{i}\left(1-a_{i}(p)\right) \alpha+b_{i i}(p) g_{i}(\beta)+P_{i}\right] \\
& \leq T M_{i} \frac{1-\hat{c}_{i}}{M_{i} T} \beta=\left(1-\hat{c}_{i}\right) \beta
\end{aligned}
$$

and

$$
\begin{aligned}
\left(C^{R} u\right)_{i}(n) & \geq \sum_{p=n}^{n+T-1} G_{i}(n, p)\left[b_{i i}(p) g_{i}(\alpha)+\sum_{j \neq i}\left|b_{i j}(p)\right| g_{j}^{\natural}-\sum_{j=1}^{m} c_{i j} \beta\left(1-a_{i}(p)\right)\right. \\
& \left.-\sum_{j=1}^{m}\left|d_{i j}(p)\right| g_{j}^{\natural}+\left|I_{i}(p)\right|\right] \\
& \geq \sum_{p=n}^{n+T-1} G_{i}(n, p)\left[-\hat{c}_{i}\left(1-a_{i}(p)\right) \beta+b_{i i}(p) g_{i}(\alpha)-P_{i}\right] \\
& \geq T m_{i} \frac{1-\hat{c}_{i}}{m_{i} T} \alpha=\left(1-\hat{c}_{i}\right) \alpha .
\end{aligned}
$$

- Case 2: As $\Sigma=L$ and $u \in \mathscr{B}^{L}, u_{i}(n) \in[-\beta,-\alpha]$ holds for each $i \in \mathcal{N}$ and all $n \in \mathbb{Z}$. It follows from $(3.1)$ and $\left(H_{2}\right)$ that

$$
\begin{aligned}
\left(C^{L} u\right)_{i}(n) & \geq \sum_{p=n}^{n+T-1} G_{i}(n, p)\left[b_{i i}(p) g_{i}(-\beta)-\sum_{j \neq i}\left|b_{i j}(p)\right| g_{j}^{\natural}-\sum_{j=1}^{m} c_{i j}(-\alpha)\left(1-a_{i}(p)\right)\right. \\
& \left.-\sum_{j=1}^{m}\left|d_{i j}(p)\right| g_{j}^{\natural}-\left|I_{i}(p)\right|\right] \\
& \geq \sum_{p=n}^{n+T-1} G_{i}(n, p)\left[\hat{c}_{i}\left(1-a_{i}(p)\right) \alpha-b_{i i}(p) g_{i}(\beta)-P_{i}\right] \\
& \geq T M_{i} \frac{1-\hat{c}_{i}}{M_{i} T}(-\beta)=-\left(1-\hat{c}_{i}\right) \beta
\end{aligned}
$$


and

$$
\begin{aligned}
\left(C^{L} u\right)_{i}(n) & \geq \sum_{p=n}^{n+T-1} G_{i}(n, p)\left[b_{i i}(p) g_{i}(-\alpha)+\sum_{j \neq i}\left|b_{i j}(p)\right| g_{j}^{\natural}-\sum_{j=1}^{m} c_{i j}(-\beta)\left(1-a_{i}(p)\right)\right. \\
& \left.+\sum_{j=1}^{m}\left|d_{i j}(p)\right| g_{j}^{\natural}-\left|I_{i}(p)\right|\right] \\
& \leq \sum_{p=n}^{n+T-1} G_{i}(n, p)\left[\hat{c}_{i}\left(1-a_{i}(p)\right) \beta-b_{i i}(p) g_{i}(\alpha)+P_{i}\right] \\
& \leq T m_{i} \frac{1-\hat{c}_{i}}{m_{i} T}(-\alpha)=-\left(1-\hat{c}_{i}\right) \alpha .
\end{aligned}
$$

It follows from above two cases about the estimation of $\left(C^{\Sigma} u\right)_{i}(n)$ that $\left\|C^{\Sigma} u\right\| \leq\left(1-\min \left\{\hat{c}_{i}\right\}\right) \beta \leq \beta$. This shows that $C^{\Sigma}(\mathscr{B} \Sigma)$ is uniformly bounded. Together with the continuity of $C^{\Sigma}$, for any bounded sequence $\left\{\psi_{n}\right\}$ in $\mathscr{B}^{\Sigma}$, we know that there exists a subsequence $\left\{\psi_{n_{k}}\right\}$ in $\mathscr{B} \sum$ such that $\left\{C^{\Sigma}\left(\psi_{n_{k}}\right)\right\}$ is convergent in $C^{\Sigma}(\mathscr{B} \Sigma)$. Therefore, $C^{\Sigma}$ is compact on $\mathscr{B}^{\Sigma}$. This completes the proof.

Theorem 3.1. Under the basic assumptions $\left(H_{1}\right)$ and $\left(H_{2}\right)$, for each $\Sigma$, (1.2) has a $T$ periodic solution $u^{\Sigma}$ satisfying $u^{\Sigma} \in \mathscr{B}^{\sum}$.

Proof. Let $u, \hat{u} \in \mathscr{B}^{\Sigma}$. We should show that $B^{\Sigma} u+C^{\Sigma} \hat{u} \in \mathscr{B}^{\Sigma}$. For simplicity we only consider the case $\Sigma=R$. It follows from (2.2) and $\left(H_{2}\right)$ that

$$
\begin{aligned}
\left(B^{R} u\right)_{i}(n) & +\left(C^{R} \hat{u}\right)_{i}(n)=\sum_{j=1}^{m} c_{i j} u_{j}(n-\tau) \\
& +\sum_{p=n}^{n+T-1} G_{i}(n, p)\left[\sum_{j=1}^{m} b_{i j}(p) g_{j}\left(\hat{u}_{j}(p)\right)-\sum_{j=1}^{m} c_{i j} \hat{u}_{j}(p-\tau)\left(1-a_{i}(p)\right)\right. \\
& \left.+\sum_{j=1}^{m} d_{i j}(p) g_{j}\left(\sum_{v=1}^{\infty} h_{j}(v) \hat{u}_{j}(p-v)\right)+I_{i}(p)\right] \\
& \leq \sum_{j=1}^{m} c_{i j} \beta+\sum_{p=n}^{n+T-1} G_{i}(n, p)\left[b_{i i}(p) g_{i}(\beta)+\sum_{j \neq 1}\left|b_{i j}(p)\right| g_{j}^{\natural}\right. \\
& \left.-\sum_{j=1}^{m} c_{i j} \alpha\left(1-a_{i}(p)\right)+\sum_{j=1}^{m}\left|d_{i j}(p)\right| g_{j}^{\natural}+\left|I_{i}(p)\right|\right] \\
& \leq \hat{c}_{i} \beta+T M_{i} \frac{1-\hat{c}_{i}}{M_{i} T} \beta=\beta .
\end{aligned}
$$

On the other hand,

$$
\begin{aligned}
\left(B^{R} u\right)_{i}(n) & +\left(C^{R} \hat{u}_{i}(n)=\sum_{j=1}^{m} c_{i j} u_{j}(n-\tau)\right. \\
& +\sum_{p=n}^{n+T-1} G_{i}(n, p)\left[\sum_{j=1}^{m} b_{i j}(p) g_{j}\left(\hat{u}_{j}(p)\right)-\sum_{j=1}^{m} c_{i j} \hat{u}_{j}(p-\tau)\left(1-a_{i}(p)\right)\right. \\
& \left.+\sum_{j=1}^{m} d_{i j}(p) g_{j}\left(\sum_{v=1}^{\infty} h_{j}(v) \hat{u}_{j}(p-v)\right)+I_{i}(p)\right] \\
& \geq \sum_{j=1}^{m} c_{i j} \alpha+\sum_{p=n}^{n+T-1} G_{i}(n, p)\left[b_{i i}(p) g_{i}(\alpha)+\sum_{j \neq 1}\left|b_{i j}(p)\right| g_{j}^{\natural}\right. \\
& \left.-\sum_{j=1}^{m} c_{i j} \beta\left(1-a_{i}(p)\right)-\sum_{j=1}^{m}\left|d_{i j}(p)\right| g_{j}^{\natural}+\left|I_{i}(p)\right|\right] \\
& \geq \hat{c}_{i} \alpha+T m_{i} \frac{1-\hat{c}_{i}}{m_{i} T} \alpha=\alpha .
\end{aligned}
$$


Therefore, all the hypotheses stated in Lemma 2.3 are satisfied. Hence, (1.2) has a $T$ periodic solution $u^{R}$ satisfying $u^{R} \in \mathscr{B}^{R}$. Almost the same argument can be done for the case $\Sigma=L$. The proof is complete.

For the case $c_{i j}<0$, we present the following assumption:

- Assumption $\left(\widehat{H}_{2}\right)$ : For each $i, j \in \mathcal{N}, c_{i j} \leq 0$ and $-1<\hat{c}_{i}:=\sum_{j=1}^{m} c_{i j}<0$. There exist constants $\alpha>0$ and $\beta>0$ with $\alpha<\beta$ such that for all $n \in \mathbb{Z}$

$$
\left\{\begin{array}{l}
\left(1-a_{i}(n)\right) \hat{c}_{i} \beta+\frac{\beta-\hat{c}_{i} \alpha}{M_{i} T}>Q_{i} \\
-\left(1-a_{i}(n)\right) \hat{c}_{i} \alpha+\frac{\hat{c}_{i} \beta-\alpha}{m_{i} T}>Q_{i} .
\end{array}\right.
$$

where

$$
Q_{i}:=\sup _{n \in \mathbb{Z}}\left\{\sum_{j=1}^{m}\left(\left|b_{i j}(n)\right|+\left|d_{i j}(n)\right|\right) g_{j}^{\natural}+\left|I_{i}(n)\right|\right\} .
$$

Similarly as Proposition 3.1, we can obtain

Proposition 3.2. Under the basic assumptions $\left(H_{1}\right)$ and $\left(\widehat{H}_{2}\right)$, for each $\Sigma$, the operator $C^{\Sigma}$ is completely continuous on $\mathscr{B}^{\Sigma}$.

Proof For any given $\Sigma$ and $u \in \mathscr{B} \Sigma$, we have two cases for the estimation of $\left(C^{\Sigma} u\right)_{i}$ (n).

- Case 1: As $\Sigma=R$ and $u \in \mathscr{B}^{R}, u_{i}(n) \in[\alpha, \beta]$ holds for each $i \in \mathcal{N}$ and all $n \in \mathbb{Z}$. It follows from $(3.1)$ and $\left(\widehat{H}_{2}\right)$ that

$$
\left(C^{R} u\right)_{i}(n) \leq \sum_{p=n}^{n+T-1} G_{i}(n, p)\left[-\sum_{j=1}^{m} c_{i j} \beta\left(1-a_{i}(p)\right)+Q_{i}\right] \leq T M_{i} \frac{\beta-\hat{c}_{i} \alpha}{M_{i} T}=\beta-\hat{c}_{i} \alpha
$$

and

$$
\left(C^{R} u\right)_{i}(n) \geq \sum_{p=n}^{n+T-1} G_{i}(n, p)\left[-\sum_{j=1}^{m} c_{i j} \alpha\left(1-a_{i}(p)\right)+Q_{i}\right] \geq T m_{i} \frac{\alpha-\hat{c}_{i} \beta}{m_{i} T}=\alpha-\hat{c}_{i} \beta .
$$

- Case 2: As $\Sigma=L$ and $u \in \mathscr{B}^{L}, u_{i}(n) \in[-\beta,-\alpha]$ holds for each $i \in \mathcal{N}$ and all $n \in$ $\mathbb{Z}$. It follows from $(3.1)$ and $\left(\widehat{H}_{2}\right)$ that

$$
\left(C^{L} u\right)_{i}(n) \geq \sum_{p=n}^{n+T-1} G_{i}(n, p)\left[-\sum_{j=1}^{m} c_{i j}(-\beta)\left(1-a_{i}(p)\right)+Q_{i}\right] \geq T M_{i} \frac{\hat{c}_{i} \alpha-\beta}{M_{i} T}=\hat{c}_{i} \alpha-\beta
$$

and

$$
\left(C^{L} u\right)_{i}(n) \leq \sum_{p=n}^{n+T-1} G_{i}(n, p)\left[-\sum_{j=1}^{m} c_{i j}(-\alpha)\left(1-a_{i}(p)\right)+Q_{i}\right] \geq T m_{i} \frac{\hat{c}_{i} \beta-\alpha}{m_{i} T}=\hat{c}_{i} \beta-\alpha .
$$


By a similar argument, we prove that $C^{\Sigma}$ is continuous and compact on $\mathscr{B}^{\sum}$. This completes the proof.

Theorem 3.2. Under the basic assumptions $\left(H_{1}\right)$ and $\left(\widehat{H}_{2}\right)$, for each $\Sigma$, (1.2) has a $T$ periodic solution $u^{\Sigma}$ satisfying $u^{\Sigma} \in \mathscr{B} \Sigma$.

Proof. Let $u, \hat{u} \in \mathscr{B}^{\Sigma}$. We should show that $B^{\Sigma} u+C^{\Sigma} \hat{u} \in \mathscr{B}^{\Sigma}$. For simplicity, we only consider the case $\Sigma=L$. It follows from $(2.2)$ and $\left(\widehat{H}_{2}\right)$ that

$$
\begin{aligned}
\left(B^{L} u\right)_{i}(n) & +\left(C^{L} \hat{u}\right)_{i}(n)=\sum_{j=1}^{m} c_{i j} u_{j}(n-\tau) \\
& +\sum_{p=n}^{n+T-1} G_{i}(n, p)\left[\sum_{j=1}^{m} b_{i j}(p) g_{j}\left(\hat{u}_{j}(p)\right)-\sum_{j=1}^{m} c_{i j} \hat{u}_{j}(p-\tau)\left(1-a_{i}(p)\right)\right. \\
& \left.+\sum_{j=1}^{m} d_{i j}(p) g_{j}\left(\sum_{v=1}^{\infty} h_{j}(v) \hat{u}_{j}(p-v)\right)+I_{i}(p)\right] \\
& \leq \sum_{j=1}^{m} c_{i j}(-\beta)+\sum_{p=n}^{n+T-1} G_{i}(n, p)\left[-\sum_{j=1}^{m} c_{i j}(-\alpha)\left(1-a_{i}(p)\right)+Q_{i}\right] \\
& \leq-\hat{c}_{i} \beta+T m_{i} \frac{\hat{c}_{i} \beta-\alpha}{m_{i} T}=-\alpha .
\end{aligned}
$$

On the other hand,

$$
\begin{aligned}
\left(B^{L} u\right)_{i}(n) & +\left(C^{L} \hat{u}\right)_{i}(n)=\sum_{j=1}^{m} c_{i j} u_{j}(n-\tau) \\
& +\sum_{p=n}^{n+T-1} G_{i}(n, p)\left[\sum_{j=1}^{m} b_{i j}(p) g_{j}\left(\hat{u}_{j}(p)\right)-\sum_{j=1}^{m} c_{i j} \hat{u}_{j}(p-\tau)\left(1-a_{i}(p)\right)\right. \\
& \left.+\sum_{j=1}^{m} d_{i j}(p) g_{j}\left(\sum_{v=1}^{\infty} h_{j}(v) \hat{u}_{j}(p-v)\right)+I_{i}(p)\right] \\
& \geq \sum_{j=1}^{m} c_{i j}(-\alpha)+\sum_{p=n}^{n+T-1} G_{i}(n, p)\left[-\sum_{j=1}^{m} c_{i j}(-\beta)\left(1-a_{i}(p)\right)+Q_{i}\right] \\
& \geq-\hat{c}_{i} \alpha+T M_{i} \frac{\hat{c}_{i} \alpha-\beta}{M_{i} T}=-\beta .
\end{aligned}
$$

Therefore, all the hypotheses stated in Lemma 2.3 are satisfied. Hence, (1.2) has a $T$ periodic solution $u^{L}$ satisfying $u^{L} \in \mathscr{B}^{L}$. By a similar argument, one can prove the case $\Sigma=R$. This completes the proof.

\section{Numerical examples}

Example 1. Consider the following neutral-type difference neural networks with delays

$$
\begin{aligned}
u_{i}(n+1)=a_{i}(n) u_{i}(n) & +\sum_{j=1}^{3} c_{i j} \Delta u_{j}(n-\tau)+\sum_{j=1}^{3} b_{i j}(n) g_{j}\left(u_{j}(n)\right) \\
& +\sum_{j=1}^{3} d_{i j}(n) g_{j}\left[\sum_{v=1}^{\infty} h_{j}(v) u_{j}(n-v)\right]+I_{i}(n),
\end{aligned}
$$


where

$$
\begin{aligned}
& a_{1}(n)=a_{2}(n)=a_{3}(n)=a_{3}(n):=\exp (-0.1-0.01 \cos 0.2 \pi n), \\
& I_{1}(n):=0.02 \cos 0.2 \pi n, I_{2}(n):=0.03 \sin 0.2 \pi n \text {, } \\
& I_{3}(n):=0.2 \sin 0.2 \pi n, \tau=5, \quad g(z):=g_{1}(z)=g_{2}(z)=\tanh (z), \quad m=3, \\
& C=\left(c_{i j}\right)=\left(\begin{array}{ccc}
0.2 & 0.1 & 0.05 \\
0.1 & 0.25 & 0 \\
0.05 & 0.1 & 0.2
\end{array}\right), h_{1}(10)=h_{2}(10)=h_{3}(10)=1, T=10 \text {, } \\
& D(n)=\left(d_{i j}(n)\right)=\left(\begin{array}{ccc}
0 & 0.05 \cos (0.2 \pi n) & 0 \\
0.1 \sin (0.2 \pi n) & 0 & 0 \\
0 & 0 & 0.01 \sin (0.2 \pi n)
\end{array}\right) \text {, } \\
& B(n)=\left(b_{i j}(n)\right)=\left(\begin{array}{ccc}
7+\sin (0.2 \pi n) & 0.1 \sin (0.2 \pi n) & 0.01 \sin (0.2 \pi n) \\
0.1 \cos (0.2 \pi n) & 7+\sin (0.2 \pi n) & 0 \\
0.01 \sin (0.2 \pi n) & 0 & 7+\sin (0.2 \pi n)
\end{array}\right) .
\end{aligned}
$$

Obviously, the sigmoidal function $\tanh (z)$ is strictly increasing on $\mathbb{R}$ with $|\tanh (z)|<$ 1. It is easy for us to check that $\left(H_{1}\right)$ holds. After some computations, we have

$$
\begin{aligned}
& \hat{c}_{1}=\hat{c}_{2}=\hat{c}_{3}=0.35, \quad m_{1}=m_{2}=m_{3}=0.6496, \\
& M_{1}=M_{2}=M_{3}=1.2720, \quad P_{1}=0.18, \quad P_{2}=0.23, P_{3}=0.22 .
\end{aligned}
$$

Take $\alpha=3, \beta=160$ and define

$$
\left.\begin{array}{l}
S_{1}(n):=\left(1-a_{i}(n)\right) \hat{c}_{1} \alpha-\left[-\frac{1-\hat{c}_{1}}{M_{i} T} \beta+b_{i i}(n) g_{i}(\beta)\right] \\
S_{2}(n):=\left[-\frac{1-\hat{c}_{i}}{m_{i} T} \alpha+b_{i i}(n) g_{i}(\alpha)\right]-\left(1-a_{i}(n)\right) \hat{c}_{i} \beta
\end{array}\right\}
$$

From Figure 1, we can check that assumption $\left(\mathrm{H}_{2}\right)$ hold. By Theorem 3.1, there exists a positive ten-periodic sequence solution of (4.1) and a negative ten-periodic sequence solution. For the coexistence of positive periodic sequence solution and its anti-sgn ones, we can refer to Figures 2 and 3. Phase view for biperiodicity dynamics of (4.1), we can refer to Figure 4.

Example 2. Consider the following neutral-type difference neural networks with delays

$$
u_{i}(n+1)=a_{i}(n) u_{i}(n)+\sum_{j=1}^{2} c_{i j} \Delta u_{j}(n-\tau)+\sum_{j=1}^{2} b_{i j}(n) g_{j}\left(u_{j}(n)\right)+I_{i}(n),
$$

where

$$
\begin{aligned}
& a_{1}(n):=\exp (-0.1-0.01 \cos 0.2 \pi n), a_{2}(n):=\exp (-0.2-0.1 \sin 0.2 \pi n), \\
& I_{1}(n):=0.02 \sin 0.2 \pi n, \quad I_{2}(n):=0.02 \cos 0.2 \pi n, \quad \tau=5, \quad g(z):=g_{1}(z)=g_{2}(z)=\tanh (z), \\
& C=\left(c_{i j}\right)=\left(\begin{array}{cc}
-0.1-0.2 \\
-0.2-0.1
\end{array}\right), T=10, \quad B(n)=\left(\begin{array}{cc}
0.5 & 0.005 \sin (0.2 \pi n) \\
0.1 \cos (0.2 \pi n) & 0.5
\end{array}\right) .
\end{aligned}
$$

Obviously, $\left(H_{1}\right)$ holds. From some computations, we have

$$
\begin{aligned}
& \hat{c}_{1}=\hat{c}_{2}=-0.3, \quad m_{1}=0.6496, \quad m_{2}=0.1912, \\
& M_{1}=1.2720, \quad M_{2}=0.8222, \quad Q_{1}=0.525, \quad Q_{2}=0.62 .
\end{aligned}
$$



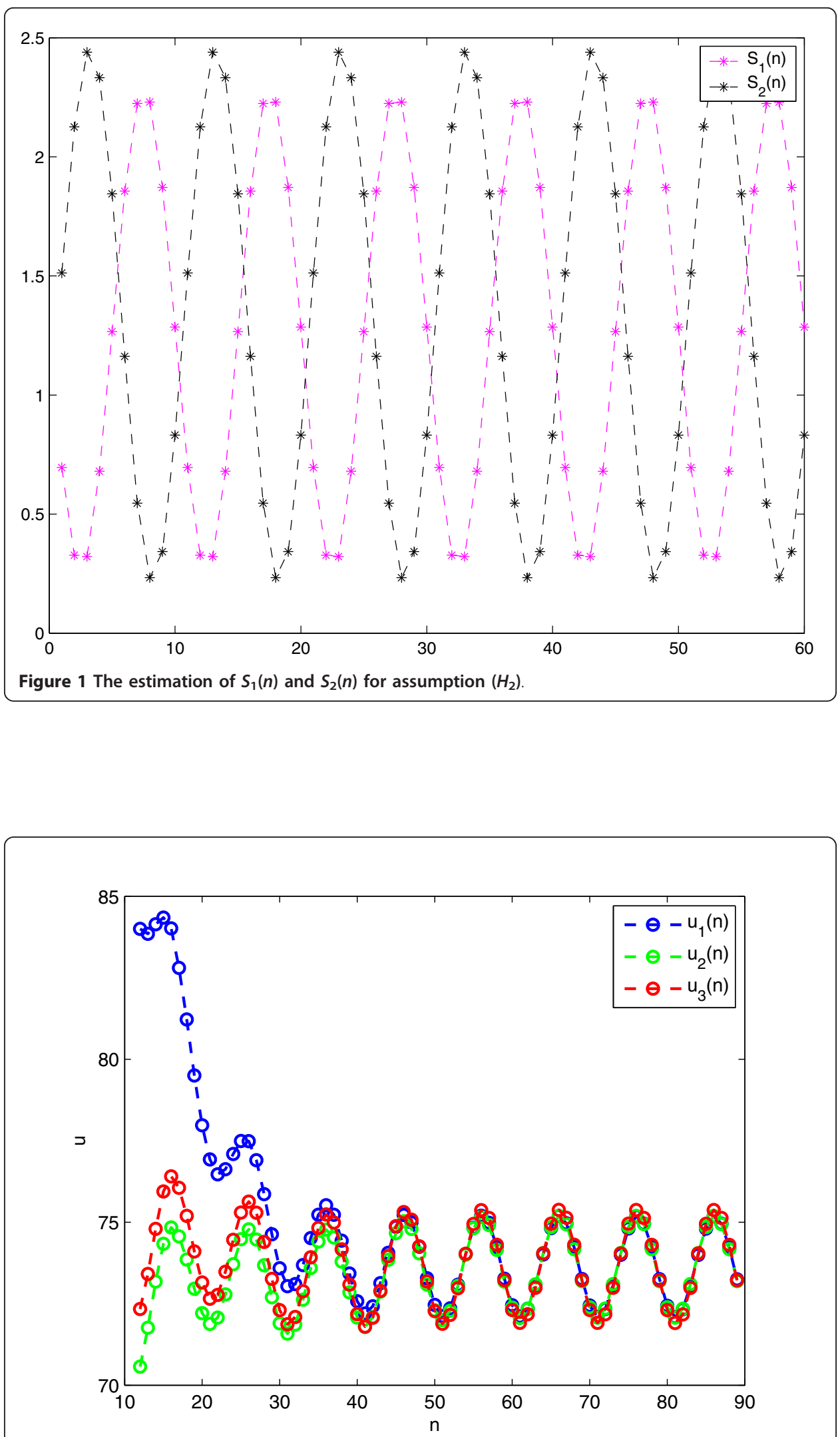

Figure 2 The existence of a positive $T$-periodic sequence solution of (4.1). 


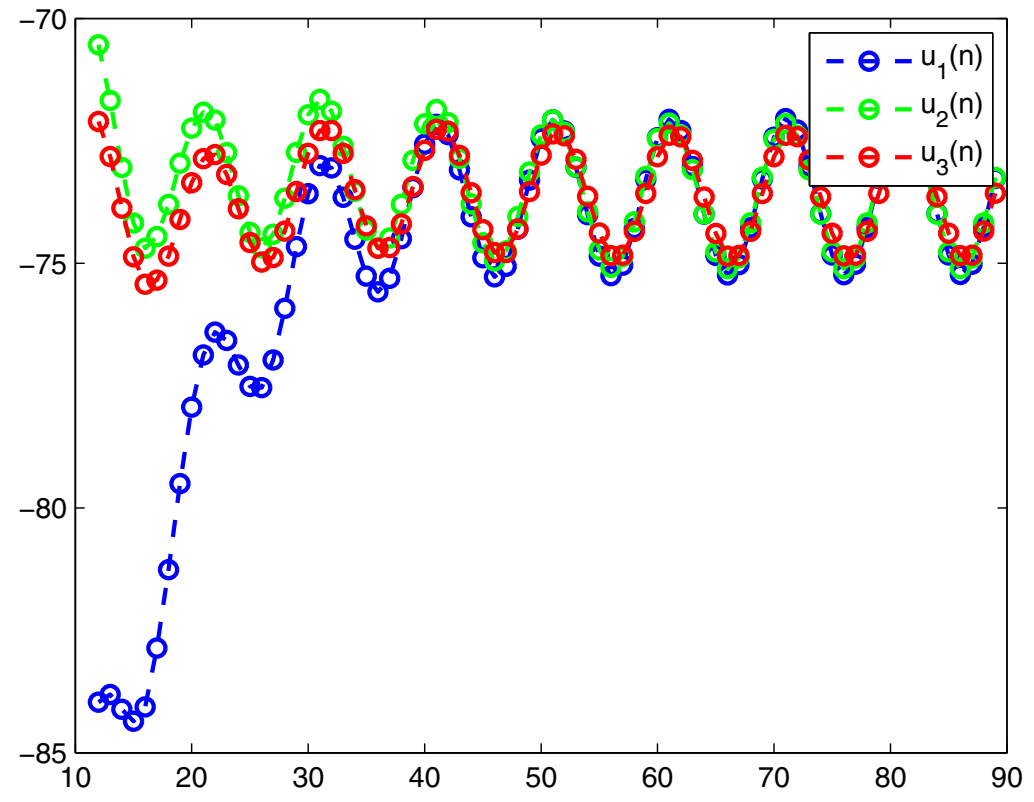

Figure 3 The existence of a negative $T$-periodic sequence solution of (4.1)

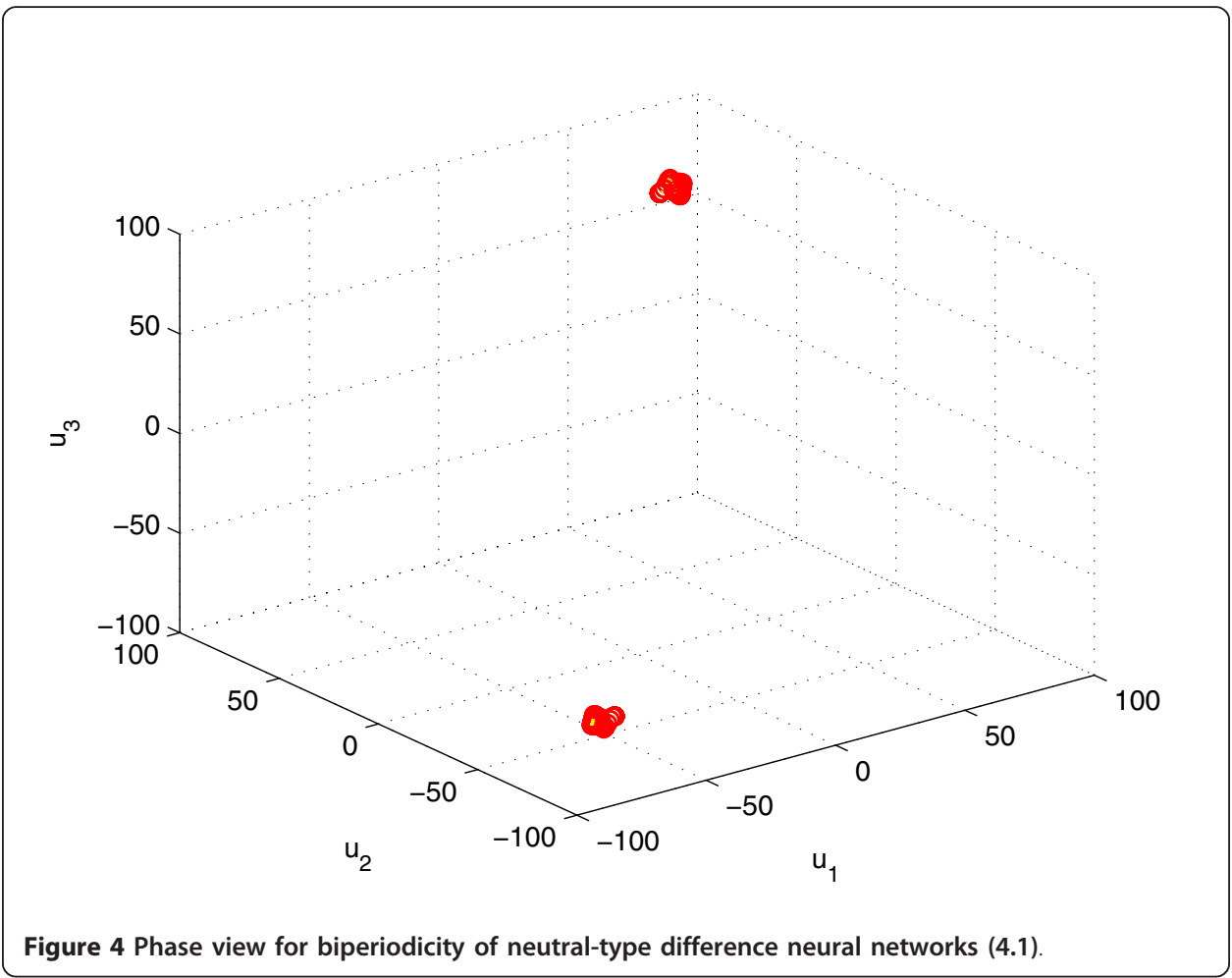


Let $\alpha=1, \beta=20$. We can check assumption $\left(\widehat{H}_{2}\right)$ holds. From Theorem 3.2, there exist a positive ten-periodic sequence solution and an anti-sgn ones of (4.2). For the coexistence of a positive $T$-periodic sequence solution and its an anti-sgn ones of (4.2), we can refer to Figure 5. Figure 6 shows phase view for biperiodicity dynamics of (4.2).

\section{Remarks and open problems}

To the best of authors' knowledge, this is the first time when biperiodicity criteria for neutral-type difference neural networks with delays

$$
\begin{aligned}
u_{i}(n+1)-a_{i}(n) u_{i}(n) & =\sum_{j=1}^{m} c_{i j} \Delta u_{j}(n-\tau)+\sum_{j=1}^{m} b_{i j}(n) g_{j}\left(u_{j}(n)\right) \\
& +\sum_{j=1}^{m} d_{i j}(n) g_{j}\left[\sum_{v=1}^{\infty} h_{j}(v) u_{j}(n-v)\right]+I_{i}(n), \quad i \in \mathcal{N}
\end{aligned}
$$

have been studied.

We propose the following open problems for future research:

Our new assumptions $\left(H_{2}\right)$ and $\left(\widehat{H}_{2}\right)$ indicate that neutral term plays an important role on the dynamics of biperiodicity. Such study has not been mentioned in the literature. However, there is still more to do. For example:

(i) If we relax the conditions $c_{i j} \leq 0$ or $c_{i j} \geq 0$ for all $i, j \in \mathcal{N}$ on the neutral term, then is the existence of multiperiodic dynamics still exist?

(ii) Evidently, in our work Biperiodicity of neural networks depends on the boundedness of activation functions. Can such requirement be relaxed and yet still obtain periodic sequence solutions and whether they are always of anti-sign?

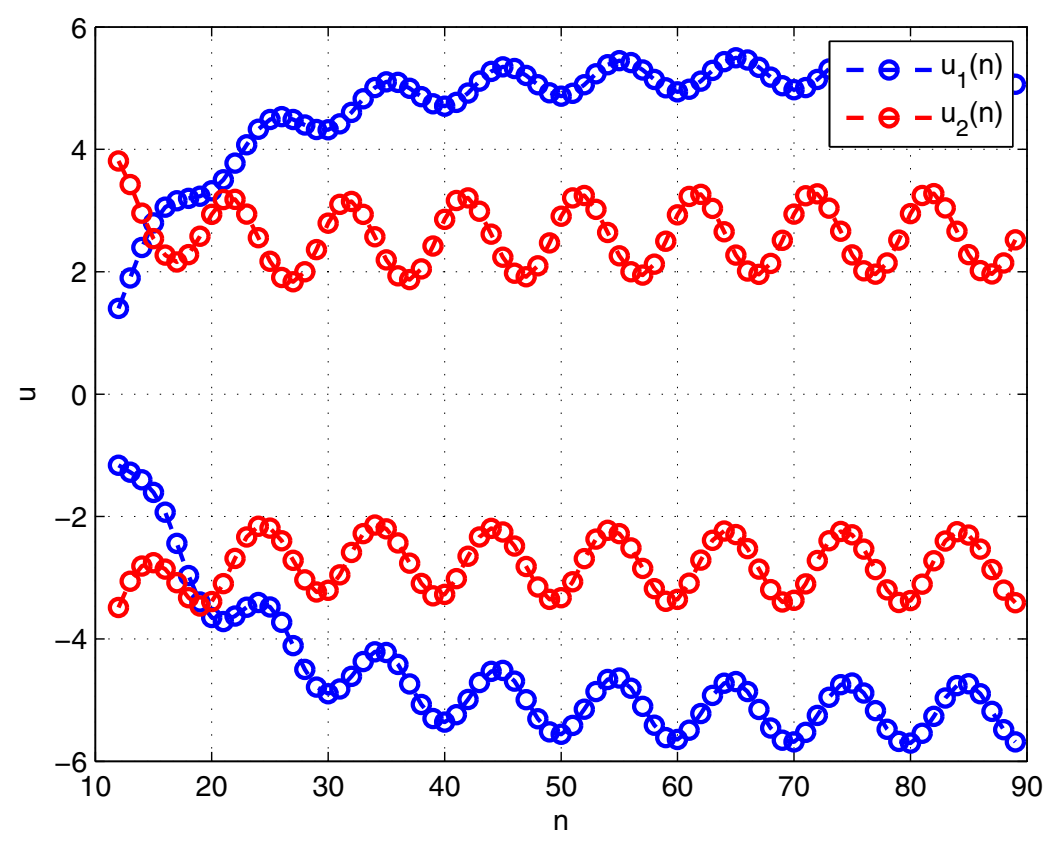

Figure 5 Coexistence of a positive $T$-periodic solution and its an anti-sgn ones of (4.2). 


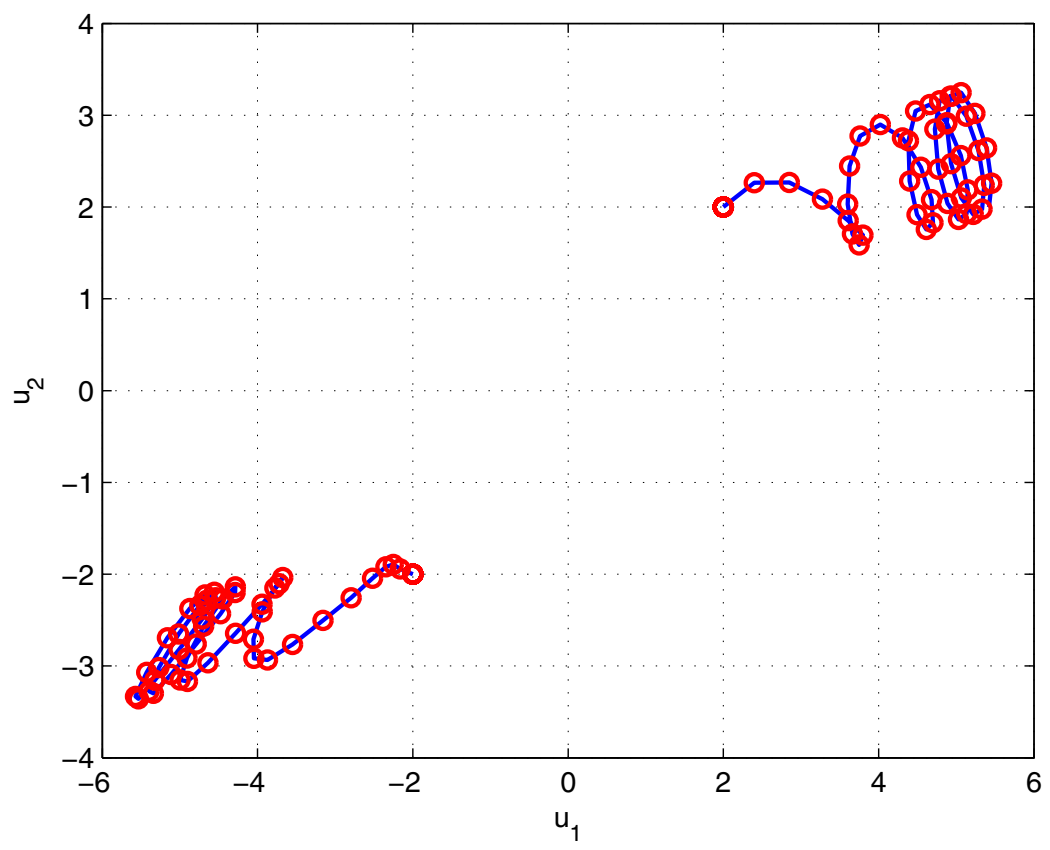

Figure 6 Phase view of biperiodicity for neutral-type difference neural networks (4.2).

To discuss the sign of each $c_{i j}$ and consider analytic properties of activation functions is a possible way to investigate these problems.

\section{Acknowledgements}

This research was supported by National Natural Science Foundation of China under Grant 11101187, the Foundation for Young Professors of Jimei University and the Foundation of Fujian Higher Education (JA10184,JA11154,JA11144).

\section{Author details}

${ }^{1}$ School of Science, Jimei University, Xiamen 361021, P. R. China ${ }^{2}$ Department of Mathematics, University of Dayton, Dayton, OH 45469-2316, USA

\section{Authors' contributions}

All the authors have contributed in all the part and they have read and approved the final manuscript.

\section{Competing interests}

The authors declare that they have no competing interests.

Received: 17 October 2011 Accepted: 31 January 2012 Published: 31 January 2012

\section{References}

1. Huang, LH, Li, XM: Dynamics of Cellular Neural Networks. Science Press, Beijing (2007)

2. Bellen, A, Guglielmi, N, Ruehli, AE: Methods for linear systems of circuit delay differential equations of neutral type. IEEE Trans Circuits Syst I Fundam Theory Appl. 46, 212-215 (1999). doi:10.1109/81.739268

3. Clarkson, ID, Goodall, DP: On the stabilizability of imperfectly known nonlinear delay systems of the neutral type. IEEE Trans Autom Control. 45, 2326-2331 (2000). doi:10.1109/9.895567

4. Zhang, HG, Liu, ZW, Huang, GB: Novel delay-dependent robust stability analysis for switched neutral-type neural networks with time-varying dalays via SC technique. IEEE Trans Syst Man, Cyber B: Cybernetics. 40, 1480-1491 (2010)

5. Park, JH, Kwon, OM, Lee, SM: LMI optimization approach on stability for delayed neural network of neutral-type. J Comput Appl Math. 196, 224-236 (2008)

6. Cheng, CJ, Liao, TL, Yan, JJ, Hwang, CC: Globally Asymptotic Stability of a Class of Neutral-Type Neural Networks With Delays. IEEE Trans Syst Man Cybern B: Cybernetics. 36, 1191-1195 (2006)

7. Samli, R, Arik, S: New results for global stability of a class of neutral-type neural systems with time delays. Appl Math Comput. 210, 564-570 (2009). doi:10.1016/.jamc.2009.01.031

8. Rakkiyappan, P, Balasubramaniam, P: New global exponential stability results for neutral type neural networks with distributed time delays. Neurocomputing. 71, 1039-1045 (2008). doi:10.1016/j.neucom.2007.11.002 
9. Kelley, W, Perterson, A: Difference Equations: An Introduction with Applications. Harcourt Acadamic Press, San Diego. (2001)

10. Chen, LN, Aihara, K: Chaos and asymptotical stability in discrete-time neural networks. Physica D: Nonlinear Phenomena. 104, 286-325 (1997). doi:10.1016/S0167-2789(96)00302-8

11. Liang, JL, Cao, JD, Ho, DWC: Discrete-time bidirectional associative memory neural networks with variable delays. Phys Lett A. 335, 226-234 (2005). doi:10.1016/j.physleta.2004.12.026

12. Liu, YR, Wang, ZD, Serrano, A, Liu, XH: Discrete-time recurrent neural networks with time-varying delays: Exponentia stability analysis. Phys Lett A. 362, 480-488 (2007). doi:10.1016/j.physleta.2006.10.073

13. Mohamad, S: Global exponential stability in continuous-time and discrete-time delayed bidirectional neural networks. Physica D. 159, 233-51 (2001). doi:10.1016/S0167-2789(01)00344-X

14. Chen, $\mathrm{WH}, \mathrm{Lu}, \mathrm{XM}$, Liang, DY: Global exponential stability for discrete-time neural networks with variable delays. Phys Lett A. 358, 186-198 (2006). doi:10.1016/j.physleta.2006.05.014

15. Brucoli, M, Carnimeo, L, Grassi, G: Discrete-time cellular neural networks for associative memories with learning and forgetting capabilities. IEEE Trans Circ Sys I. 42, 396-399 (1995). doi:10.1109/81.401156

16. Wang, L, Zou, X: Capacity of stable periodic solutions in discrete-time bidirectional associative memory neural networks. IEEE Trans Circ Syst II. 51, 315-319 (2004). doi:10.1109/TCSII.2004.829571

17. Zeng, ZG, Wang, J: Multiperiodicity of discrete-time delayed neural networks evoked by periodic external inputs. IEEE Trans Neural Netw. 17, 1141-1151 (2006). doi:10.1109/TNN.2006.877533

18. Zhao, HY, Sun, L, Wang, GL: Periodic oscillation of discrete-time bidirectional associative memory neural networks. Neurocomputing. 70, 2924-2930 (2007). doi:10.1016/..neucom.2006.11.010

19. Zou, L, Zhou, Z: Periodic solutions for nonautonomous discrete-time neural networks. Appl Math Lett. 19, 174-185 (2006). doi:10.1016/j.aml.2005.05.004

20. Zhou, Z, Wu, JH: Stable periodic orbits in nonlinear discrete-time neural networks with delayed feedback. Comput Math Appl. 45, 935-942 (2003). doi:10.1016/S0898-1221(03)00066-X

21. Huang, ZK, Wang, XH, Gao, F: The existence and global attractivity of almost periodic sequence solution of discretetime neural networks. Phys Lett A. 350, 182-191 (2006). doi:10.1016/j.physleta.2005.10.022

22. Raffoul, Y: Periodic solutions for scalar and vector nonlinear difference equations. Panamer J Math. 9, 97-111 (1999)

23. Raffoul, Y, Yankson, E: Positive periodic solutions in neutral delay difference equations. Adv Dyn Syst Appl. 5, 123-130 (2010)

doi:10.1186/1687-1847-2012-5

Cite this article as: Huang and Raffoul: Biperiodicity in neutral-type delayed difference neural networks. Advances in Difference Equations 2012 2012:5.

\section{Submit your manuscript to a SpringerOpen ${ }^{\ominus}$ journal and benefit from:}

- Convenient online submission

- Rigorous peer review

- Immediate publication on acceptance

- Open access: articles freely available online

- High visibility within the field

- Retaining the copyright to your article

Submit your next manuscript at $\boldsymbol{\wedge}$ springeropen.com 